\title{
Keypoints Selection in the Gauss Laguerre Transformed Domain
}

\author{
Lorenzo Sorgi ${ }^{1}$, Nicola Cimminiello ${ }^{1}$ and Alessandro Neri ${ }^{2}$ \\ 1.sorgiecira.it nerieuniroma3.it \\ ${ }^{1}$ Virtual Reality Lab. - Italian Aerospace Research Centre \\ V. Maiorise sn, 81043 Capua (CE), Italy \\ 2 Dept. Applied Electronics - University of Roma TRE \\ via della Vasca Navale 84, 00146 Rome, Italy
}

\begin{abstract}
The present paper is devoted to the introduction of a novel technique to select keypoints from digital images and build representative and distinctive descriptors. The algorithm performs a multiresolution image analysis in the Laguerre Gauss transformed domain and collects in a local descriptor the transformed coefficients at multiple scales, of the keypoint's representative pattern. The rotation invariance of the Circular Harmonics and the multiscale approach make the system more robust than other descriptors to match patterns related by affine transformations.
\end{abstract}

\section{Introduction}

The extraction of distinctive keypoints from digital images is a low level vision procedure widely used in many computer vision tasks. Uncalibrated stereo matching $[7,8]$, object recognition [9] and building panoramas [1] represents just a small sample among a large set of possible applications. The history of image feature selection dates back to 1988 when Harris and Stephens presented their corner detector algorithm [2]; since then many different approaches to select keypoints and match corresponding features extracted from multiple views, have been presented. For an exhaustive review of the literature on this topic we refer to [5]. The invariance with respect to a class of projective and photometric transformations as wide as possible is the desired property for keypoints descriptors, in order to achieve a robust and reliable matching across multiple views.

In this paper we present a novel technique of keypoint selection based on the theory of Circular Harmonics Functions (CHF), which has been already introduced in the last decade in the field of pattern recognition [6]. CHFs are complex, polar separable filters characterised by harmonic angular shape, a useful property to build rotationally invariant descriptors. A scale parameter is also introduced to perform a multiresolution analysis.

CHFs with different radial profile have been studied in literature. In this context we will mainly focus on the Gauss-Laguerre CHFs which constitute a family of orthogonal functions satisfying the wavelet admissibility condition required for multiresolution wavelet pyramid. In essence, any image may be represented by translated, dilated and rotated 
replicas of any Gauss-Laguerre function. Also for a fixed resolution, the Gauss-Laguerre CHFs provide a local representation of the image in a polar coordinates system centred at a given point, named pivot. This representation is called the Gauss-Laguerre transform.

The work will present the multiresolution technique used to identify the keypoints in the image and to construct the representative descriptors by collecting a subset of the corresponding pattern transformed coefficients. The effectiveness of the algorithm, has been tested by matching some sample frames selected from image sequences. The dataset, collecting the frame sequence and the relating homographies, is available on the web. For a complete comparison with the state of the art we refer to [5] where the same dataset has been used; in this paper we present only an performance evaluation compared with SIFT [4], one of the most popular algorithms.

\section{Mathematical preliminaries}

A Circular Harmonic Function (CHF) is a complex polar separable filter characterised by a harmonic angular shape, which is represented in polar coordinates as:

$$
f^{n}(\rho, \theta)=V^{n}(\rho) e^{-j n \theta}
$$

where $n$ is the order of the CHF and $V^{n}(\rho)$ is the radial profile. Given an image $I \in$ $L^{2}\left(\mathbb{R}^{2}, d x^{2}\right)$, let $I_{p}(\rho, \theta)=I(\bar{x}+\rho \sin \theta, \bar{y}+\rho \cos \theta)$ be its representation in the polar coordinates space centred at the pivot $\mathbf{x}=(\bar{x}, \bar{y})$. Since $I_{p}$ is periodic with respect to $\theta$ it can be decomposed in terms of CHF:

$$
I_{p}(\rho, \theta)=\sum_{n} V^{n}(\rho) e^{j n \theta}
$$

where $V^{n}(\rho)$ is the radial profile corresponding to the n-th $\mathrm{CH}$ of $I_{p}(\rho, \theta)$, given by the Fourier integral:

$$
V^{n}(\rho)=\frac{1}{2 \pi} \int_{0}^{2 \pi} I_{p}(\rho, \theta) e^{-j n \theta} d \theta .
$$

With this formulation the radial profile depends on the actual image. A more general representation is obtained by expanding the radial profiles as series of weighted orthogonal functions. For the image inspection here proposed we considered two different CHFs families: those with Laguerre Gauss (LG) radial profile [6] and the Zernike moments [10, 3](Fig. 1).

The Laguerre Gauss CHF are defined as:

$$
\mathscr{L}_{n}^{k}(\rho, \theta)=(-1)^{k} 2^{(|n|+1) / 2} \pi^{|n| / 2}\left[\frac{k !}{(|n|+k) !}^{1 / 2}\right] \rho^{|n|} L_{k}^{n}\left(2 \pi \rho^{2}\right) e^{-\pi \rho^{2}} e^{j n \theta},
$$

where $L_{k}^{n}(\rho)$ are the generalized Laguerre polynomials defined by the Rodrigues formula:

$$
L_{k}^{n}(\rho)=\sum_{h=0}^{k}(-1)^{h}\left(\begin{array}{l}
n+k \\
k-h
\end{array}\right) \frac{\rho^{h}}{h !} .
$$


Zernike polynomials, first proposed in 1934 by Zernike [11], form a set of complex and orthogonal polynomials, defined over the interior of a unit circle $\rho \leq 1$ :

$$
Z_{k}^{n}(\rho, \theta)=\sum_{h=0}^{\frac{k-|n|}{2}}(-1)^{h} \frac{(k-h) !}{h !\left(\frac{k+|n|}{2}-h\right) !\left(\frac{k+|n|}{2}+h\right) !} \rho^{k-2 h} e^{j n \theta}
$$

where $k$ is a non-negative integer and $n$ is an integer such that $k-|n|$ is even and $|n|<k$.
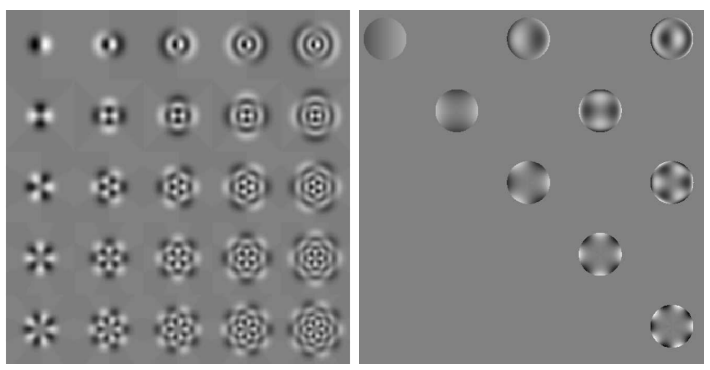

Figure 1: (a) Laguerre Gauss CHFs and (b) Zernike CHFs.

The harmonic angular shape of CHFs implies that any n-order CHF can be rotated by means of a simple phase shift:

$$
f_{k}^{n}(\rho, \theta-\phi)=V_{k}^{n}(\rho) e^{j n \theta} e^{-j n \phi} .
$$

As demonstrated in [6] any CHF of the form (1) defines a Circular Harmonic Wavelet. This means that an image $I(x, y)$ may be represented by its continuous wavelet transform $W_{f_{k}^{n}}[I](b, \alpha, \sigma)$, where $b, \alpha$ and $\theta$ are the parameters representing respectively the translated, rotated and scaled version of the mother wavelet $f_{k}^{n}(x, y)$. Also CH Filters may be successfully used for many low level vision tasks, thanks to their selectivity with respect to the basic patterns. CHF with different order are indeed natural detectors for different classes of features: CHFs of order $n=1$ for example are tuned on the borders, $n=2$ to lines, $n=3$ to three-lines corners, etc. (Fig. 2.c).
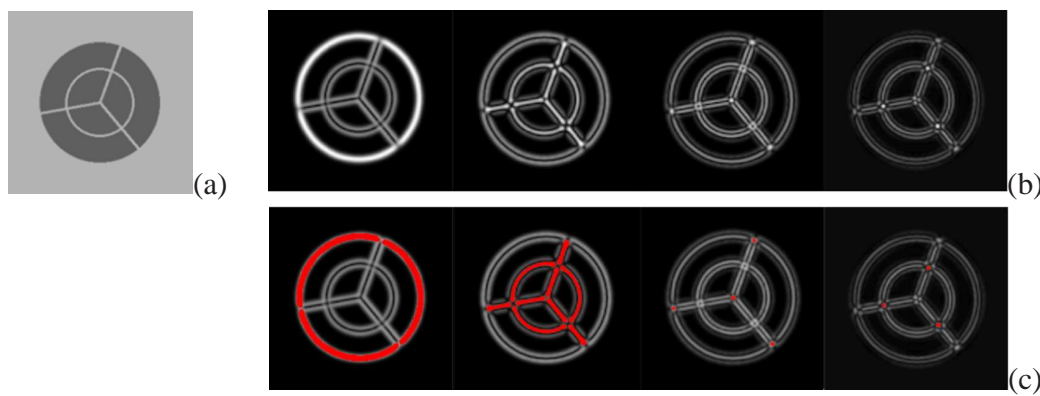

Figure 2: (a) Reference pattern; (b) Filtering response to CHFs of order $n=1, \ldots, 4$; (c) Thresholded output which identify the selected basic patterns. 


\section{Keypoints Selection}

The image projection onto the CHF basis is performed by the convolution operator:

$$
\hat{I}_{k}^{n}(x, y)=I(x, y) * f_{k}^{n}(x, y) .
$$

The self-steerability property of the CHFs implies that a 2D rotation around the pivot $\mathbf{x}=(\bar{x}, \bar{y})$ by an angle $\phi$ produces a phase shift of the projected image coefficients $\hat{I}_{k}^{n}(\mathbf{x})$ :

$$
\hat{I}_{r k}^{n}(\bar{x}, \bar{y})=\hat{I}_{k}^{n}(\bar{x}, \bar{y}) e^{-j n \phi}
$$

where $I_{r}(\mathbf{x})$ is the rotated image $I_{r}(\rho, \theta)=I(\rho, \theta-\phi)$. This interesting property will be used to build rotation invariant keypoint descriptors as detailed in the next section.

In order to perform a multiscale image analysis and to detect keypoints in a multiresolution fashion, we consider a bank of CHFs at different scales:

$$
f_{k}^{n}(\mathbf{x}, \sigma)=V_{k}^{n}\left(\frac{\rho}{\sigma}\right) e^{-j n \theta},
$$

Let $\sigma$ be the scale parameter, ranging within a bounded interval $\sigma \in\left[2^{-s_{\max }} ; 2^{s_{\max }}\right]$, discretized in $\left(2 \cdot S_{\max }+1\right)$ octaves, each one collecting $N_{s}$ uniformly sampled scales. We found experimentally that $s_{\max }=3$ and $N_{s}=5$ provide a reasonable trade-off between the accuracy and computational cost. Taking advantage of the CHFs property of being natural detectors of different classes of basic pattern, we are able to associate the keypoints with some particular image feature: lines and borders may lead to the selection of a huge amount of points, complex structures instead, may be unstable and noise sensitive. For this reason we focused on triades and corners, which are detected by inspecting the image with a compact filters bank collecting, for any possible scale, two different CHF filters, tuned to the two former classes of image features respectivelyThus the filters is a collection of pairs of CHF filters of order 3 and 4 and degree 0 , $B F=\left\{f_{k}^{n}(\mathbf{x}, \sigma), n=3,4 ; k=0 ; \sigma \in\left[2^{-s_{\max }} ; 2^{s_{\max }}\right]\right\}$ (Fig. 3.a).

The orthogonality of CHFs allows us to compute $2 N_{s}\left(S_{\text {max }}+1\right)$ energy maps of filtered images, by pair wise combining the outputs of the filter bank:

$$
S(x, y, \sigma)=\left|\hat{I}_{0}^{3}(x, y, \sigma)\right|^{2}+\left|\hat{I}_{0}^{4}(x, y, \sigma)\right|^{2} .
$$

Such an energy function $S(x, y, \sigma)$ is refereed to as image scalogram. The scalogram inspection is performed by means of a $3 \mathrm{D}$ slighting window of size $(5 \times 5 \times 3)$ pixels, in order to detect the energy local maxima, whose position are selected as potential keypoints. These are identified by the triplets $\bar{K} \triangleq(\bar{x}, \bar{y}, \bar{\sigma})$, where $(\bar{x}, \bar{y})$ are the kepoint image coordinates and $\bar{\sigma}$ will be denoted in the following as the reference scale. During the scalogram inspection we also consider the number of potential keypoints insisting to the same image location, that is the number of different reference scales associated with the same image location. To improve the keypoints stability we allow a generic position $\overline{\mathbf{x}}=(\bar{x}, \bar{y})$ to be associated to one or at most two different reference scales, according to the scheme shown in Fig. 3.b. Locations with more than two representative scales are rejected as unstable keypoints. This step usually produces a large number of candidates, 


\section{CH Filter Bank}

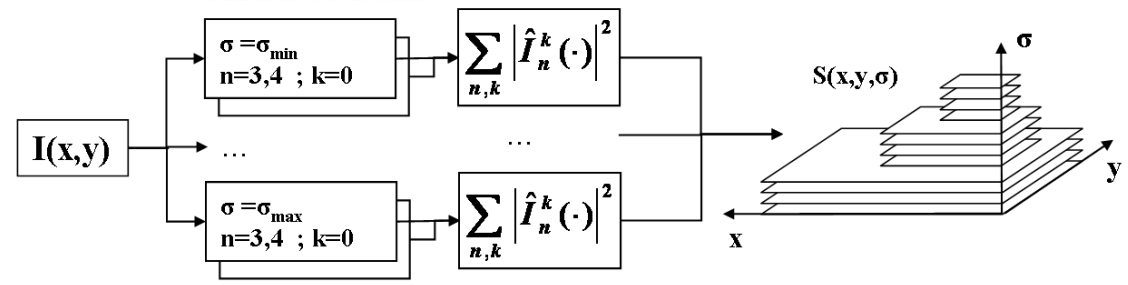

(a)
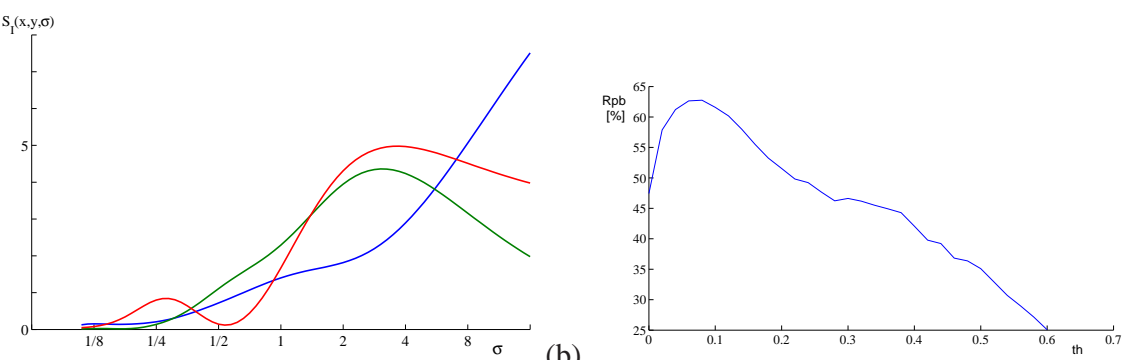

(c)

Figure 3: (a) Scheme of the scalogram construction procedure. (b) Keypoint candidates selection. Green line: one candidate; Red line: two candidates; Blue line: no candidates. (c) Repeatability test used to select the threshold gain $t h$.

which is further reduced by rejecting those keypoints $\bar{K}$ whose energy value $S(\bar{x}, \bar{y}, \bar{\sigma})$ does not exceed an adaptive threshold:

$$
S(\bar{x}, \bar{y}, \bar{\sigma})<t h \cdot \max _{x, y}(S(x, y, \bar{\sigma})) .
$$

The threshold gain th is chosen to maximize the repeatability index defined as the percentage of common keypoints selected in a pair of views (see Fig. 3.c).

\section{Keypoints Descriptors}

A generic keypoint $\bar{K}=(\bar{x}, \bar{y}, \bar{\sigma})$ is associated to a local descriptor $\bar{\chi}=\{\bar{\chi}(n, k, i), n=$ $\left.1, \ldots, n_{\max }, k=0, \ldots, k_{\max }, i=-2, \ldots, 2\right\}$. This is a normalized complex-valued vector collecting the image projection onto a wider set of CHFs, at the scales neighbour of the reference scale $\bar{\sigma}$, whose elements are given by:

$$
\bar{\chi}(n, k, i)=C_{n o r m} \cdot \hat{I}_{k}^{n}\left(\bar{x}, \bar{y}, \sigma_{i}\right) e^{-j n \phi_{i}} .
$$

Notice that with an abuse of notation $\sigma_{i}$ denotes the i-th scale following $\bar{\sigma}$ if $i>0$, or preceding $\bar{\sigma}$ if $i<0$, in the discretized scale space.

The normalization coefficient $C_{n o r m}$ is simply the inverse of the descriptor norm. Unitnorm descriptors are in fact more robust against illumination changes. Finally the phase shift $e^{-j n \phi_{i}}$ is introduced in order to make the descriptors invariant with respect to the 
keypoint pattern orientation. This property is obtained by neutralizing the angle of the transformed coefficient of degree 1 and order 0 , at scale $\sigma_{i}$ :

$$
\phi_{i}=\angle\left(\hat{I}_{0}^{1}\left(\bar{x}, \bar{y}, \sigma_{i}\right)\right)
$$

where $\angle($.$) denotes the phase of a complex number. Therefore a different phase com-$ pensation is applied to the five portions of the descriptor corresponding to the subset of normalized transformed coefficients at scale $\sigma_{i}$. It can be easily shown that rotated patterns produce identical normalized phase-compensated descriptors.

Let $\chi$ and $\chi_{\mathbf{r}}$ be the unit-norm descriptors extracted respectively from a given keypoint patch $T(\mathbf{x})$ and from the same pattern rotated about its centre $\mathbf{x}_{\mathbf{c}}=(\bar{x}, \bar{y})$ by an angle $\alpha$. The self-steerability property (9) of the pattern $\mathrm{CH}$ transformed coefficients implies that under a 2D rotation the absolute value of the descriptors elements holds unchanged:

$$
\left|\chi_{\mathbf{r}}\left(n, k, \sigma_{i}\right)\right|=\left|\chi\left(n, k, \sigma_{i}\right)\right| . \quad \forall n, k, i,
$$

The phase of any element of $\chi_{\mathbf{r}}$ is given by:

$$
\begin{aligned}
\angle \chi_{\mathbf{r}}\left(n, k, \sigma_{i}\right) & =\angle\left(\hat{T}_{r k}^{n}\left(\bar{x}, \bar{y}, \sigma_{i}\right)\right)-n \phi_{i}=\angle\left(\hat{T}_{r k}^{n}\left(\bar{x}, \bar{y}, \sigma_{i}\right)\right)-n \angle\left(\hat{T}_{r 0}^{1}\left(\bar{x}, \bar{y}, \sigma_{i}\right)\right)= \\
& =\angle\left(\hat{T}_{k}^{n}\left(\bar{x}, \bar{y}, \sigma_{i}\right)\right)-n \alpha-n\left(\angle\left(\hat{T}_{0}^{1}\left(\bar{x}, \bar{y}, \sigma_{i}\right)\right)-\alpha\right)= \\
& =\angle\left(\hat{T}_{k}^{n}\left(\bar{x}, \bar{y}, \sigma_{i}\right)\right)-n \angle\left(\hat{T}_{0}^{1}\left(\bar{x}, \bar{y}, \sigma_{i}\right)\right)=\angle \chi\left(n, k, \sigma_{i}\right) .
\end{aligned}
$$

The last term represents the phase of the normalized descriptor extracted from the unrotated patch. Therefore we can state that rotated versions of the same pattern produce identical normalized phase-compensated descriptors. Similarly we can imagine that a given patch arbitrarily transformed by an affine transformation and then rotated around its centre by an angle $-\phi_{i}$ will (hopefully) appear with the same orientation (Fig. 4.a).
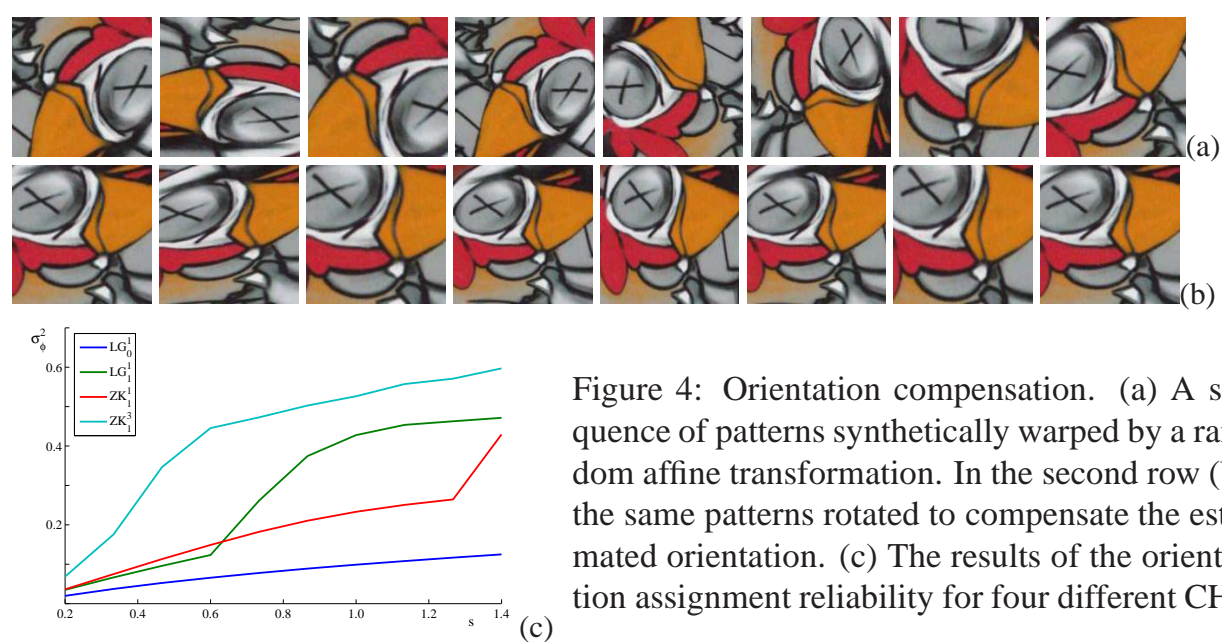

Figure 4: Orientation compensation. (a) A sequence of patterns synthetically warped by a random affine transformation. In the second row (b) the same patterns rotated to compensate the estimated orientation. (c) The results of the orienta(c) tion assignment reliability for four different $\mathrm{CH}$.

The robustness of this orientation assignment has been evaluated by transforming a set of a hundred test keypoints $K_{j}$ by a progressive scaling and then computing the variance 
of the orientation angle estimates. The scaling parameters $s_{x}$ and $s_{y}$ have been extracted as random independent variables uniformly distributed in the interval $s_{x}, s_{y} \in\left[\frac{1}{1+s}, 1+s\right]$, whose width is controlled by a unique parameter $s$. The latter is progressively incremented from 0.2 up to 1.4 and for each pair $\left(s_{j}, K_{j}\right) 100$ different tests have been performed. The variance of the orientation estimates has been taken as a measure of the algorithm reliability. In Fig. 4.c the results obtained by using four different CHFs are shown. Since the Laguerre Gauss CHF $L_{0}^{1}(x, y)$ gives the best result, we decided to use CHFs from this family to select the image keypoints and to build the local keypoints descriptors.

\section{Results}

The algorithm performance has been evaluated by matching real images selected from a dataset freely available on the web, which provides the image and the relating homographies sequences (http: //www.robots.ox.ac.uk/vgg/research/affine/). The threshold (3) which controls the number of selected keypoints has been set to $t h=$ 0.15 ; such a value in fact, has been empirically identified as the one which maximizes the repeatability test. The latter has been performed on a set of 50 image pairs synthetically generated by applying a random projectivity to 50 single still images (see Fig. 3.c). The keypoints descriptors have been constructed using the Laguerre Gauss CHF of order $n=1, \ldots, 5$ and degree $k=0, \ldots, 4$, at 5 different scales (the keypoint reference scale and its four neighbours). This leads to descriptors arrays of 125 elements which turned out to be an effective trade off between the computational cost and matching effectiveness. For comparison purpose we have run the same test with the SIFT algorithm as well, which is probably one of the most effective among those presented in the recent literature on keypoints selection. We acknowledge the author for kindly proving the algorithm code on his home-page (http: / /www.cs.ubc.ca/ lowe/keypoints/).

In the dataset we selected several image pairs from three sequences, BARK, BOAT and $G R A F$ respectively. The first two sequences are related by rotation and zoom. The obtained results show how the Laguerre Gauss CHFs seems to provide a more robust representation of templates and guarantees quite fair detection rate for images related by this class of transformation (Fig. 5.a, 5.b). This is a natural consequence of the CHFs rotational invariance, whose effectiveness for the orientation assignment has been evaluated for four different CHFs (see Fig. 4.c). The reliability of this assignment and the light computational cost due only to a single filtering operation, makes this procedure suitable for any application where the assignment of an orientation to a given pattern is required.

The last tests are selected from a sequence where the viewpoint progressively changes. In this case presenting a complete projectivity, the results show how a distribution based algorithm as SIFT, progressively outperforms with respect to a filtering based algorithm as the one presented in this work. 


\section{Conclusions}

In this work a multiscale technique for keypoints selection and matching have been illustrated. For any processing step the same inspection kernel has been used, namely a multiresolution bank of Circular Harmonic Filters, which turned out to be a fast and reliable framework to identify image keypoints and construct representative descriptors. The presented preliminary results show how the usage of such a class of inspection filters is a promising technique which performs very well in presence of scaling and rotations. The procedure will be further investigated, by analyzing the possibility of making the descriptors invariant to a general affine transformation. The property of CHFs of being natural detector for specific image primitives will be used in future work to derive a shape normalization procedure.

\section{References}

[1] M. Brown and D. G. Lowe. Recognising panoramas. In IEEE 9th International Conference on Computer Vision, pages 1218-1225, 2003.

[2] C. G. Harris and M. Stephens. A combined corner and edge detector. In In Proc. 4th Alvey Vision Conf., Manchester., pages 147-151, 1998.

[3] H. Hse and A.R. Newton. Sketched symbol recognition using zernike moments. 17th International Conference on Pattern Recognition, 1:23-26, 2004.

[4] D. Lowe. Distinctive image features from scale-invariant keypoints. International Journal of Computer Vision., pages 91-110, 2004.

[5] K. Mikolajczyk and C. Schmid. A performance evaluation of local descriptors. PAMI, 2004.

[6] A. Neri and G. Iacovitti. Maximum likelihood localization of 2-d patterns in the gauss-laguerre transform domain: Theoretic framework and preliminary results. IEEE Transaction on Image Processing, 13:72-86, 2004.

[7] F. Schaffalitzky and A. Zisserman. Multi-view matching for unordered image sets. In European Conference on Computer Vision, pages 414-431, 2002.

[8] Tinne Tuytelaars and Luc Van Gool. Matching widely separated views based on affine invariant regions. Int. J. Comput. Vision, 59(1):61-85, 2004.

[9] Tinne Tuytelaars Vittorio Ferrari and Luc Van Gool. Simultaneous object recognition and segmentation from single or multiple model views. International Journal of Computer Vision, January 2006.

[10] A. Wallin and O. Kubler. Complete sets of complex zernike moment invariants and the role of the pseudoinvariants. IEEE Transaction Pat. An. and Machine Intell., 17:1106-1110, 1995.

[11] F. Zernike. Beugungstheorie des schneidenverfahrens und seiner verbesserten form, der phasenkontrastmethode. Physica, 1:689-704, 1934. 

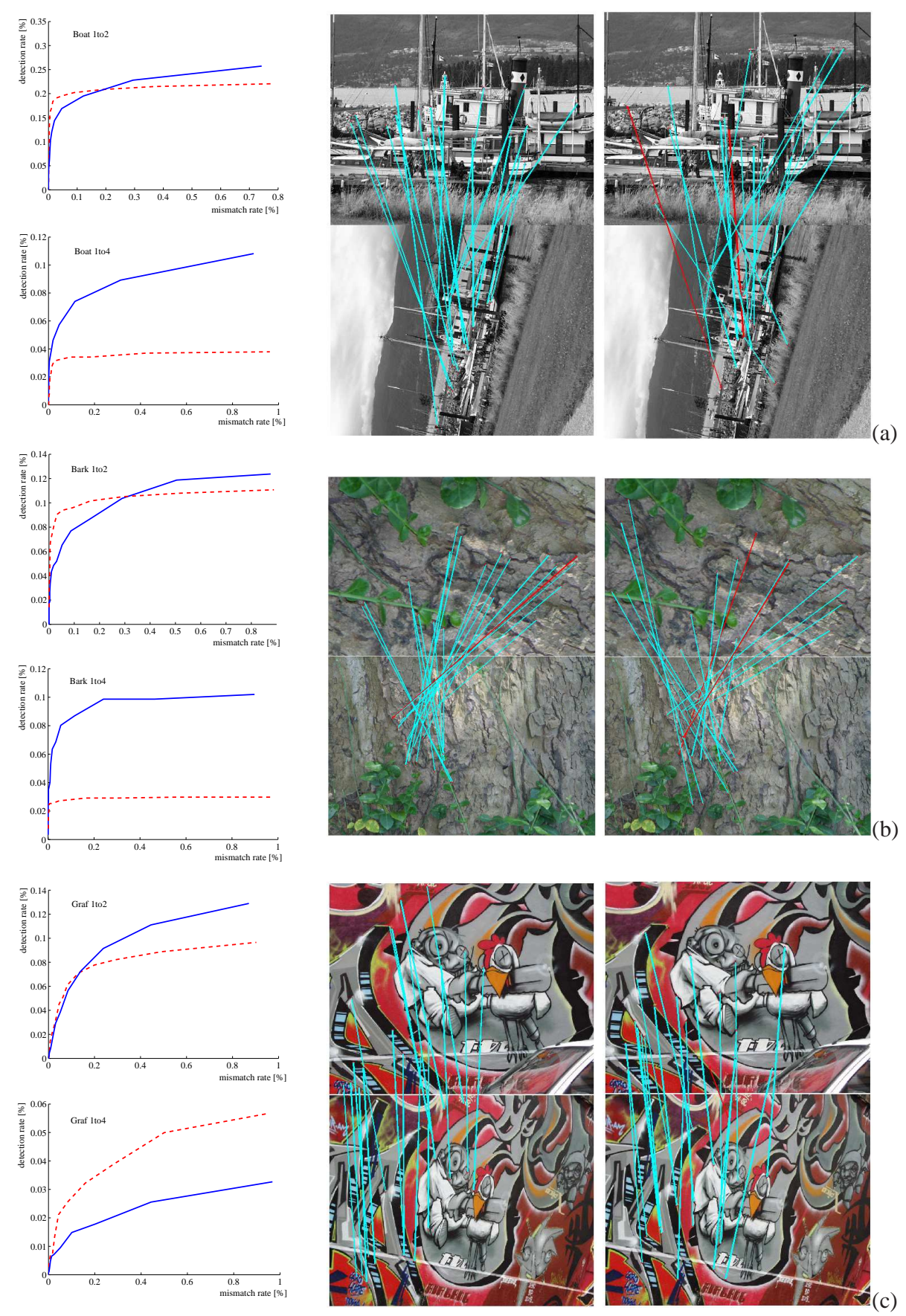

Figure 5: Matching results. On the left column the ROC curves obtained by using the LG keypoints descriptors (blue line) and SIFT (red dashed line), on the image pairs Boat 1to2, Boat1to4, Bark1to2, Bark1to4, Graf1to2 and Graf1to4. The first twenty best matches selected from the pairs Boat 1to4, Bark1to4 and Graf 1to 4 using the proposed algorithm and SIFT are shown in the central and right column respectively. 